\title{
*THE USE OF DIPHTHERIA ANTITOXIN AND TOXOID IN LEPROSY
}

\section{A Preliminary Report}

D. R. Collier and J. Hugh McKian.

\section{INTRODUCTION}

Following the encouraging results of experimental work on infecting monkeys with leprosy following a diet of colocasia, and the use of diathermy in stimulating adrenal glands in lepers, reports of which are being published at the same time as this article, Oberdoerffer and Collier decided to try the use of diphtheria antitoxin. The reasons are as follows: Oberdoerffer has advanced the theory that adrenal insufficiency predisposes an individual to leprosy. This may be caused by the sapotoxins in certain food plants, as well as result from other causes of glandular hypo-function. We thought, that there might be something more than an analogy between the well proven attempt of the body to form antibodies against a toxin such as diphtheria toxin, which essentially damages the adrenals, and the lack of such an attempt in leprosy.

* Reprinted with the permission of the authors from the "Thai Science" Bulletin II, 2, April, I940. The plates appearing in the original paper are absent, but two photographs sent by the authors show the effects of toxoid treatment on a patient not specially mentioned in the text. 
We did not expect that diphtheria antitoxin would cure leprosy, but it might be that diphtheria antitoxin would neutralize leprosy toxin in the toxaemic syndrome in leprosy, namely leprous reaction. As far as we have been able to determine the literature contains only one reference to the use of antitoxin in leprosy. Babes treated lepromatous lepers ( 2 cases) with diphtheria antitoxin and reported initial flattening out of nodules, but he never wrote on the subject again. It is too early to state definitely the kind of chemical relation or biological relation between leprosy and diphtheria, but the use of antitoxin and toxoid, as shown in the following report, does show results which do not seem to be that of a nonspecific serum protein.

\section{EXPERIMENTS WITH DIPHTIIERIA ANTITOXIN}

On December 6th, I938, we chose a number of patients who were having repeated leper reactions. As controls a number of similar cases were given injections of tetanus antitoxin, and several others were given antivenom. Approximately forty injections of these two substances were given. Case histories of a number of patients treated with diphtheria antitoxin are given below. In general the effects have been as follows: after the initial injection of 6,000 units of diphtheria antitoxin in a case of acute reaction, the patient may return the next day complaining that his fever has increased and that he is worse than before. After three or four days he begins to improve. In many cases the leprosy nodules and elevated red areas scale over, that is a fine, whitish, rather transparent membrane forms over the area. This is followed by a gradual shrinking of the area, and a change in colour from bright red to a dark brown. In the cases of more acute reaction the centre of the nodule may soften with the accumulation of a purulent fluid in the centre. If this fluid is examined it will be found to contain acid-fast bacilli of the morphology of the Microbacterium leprae.

A definite change in the form and shape of the bacteria occurs. as far as our records show, in every case where they are to be found at all. At first the bacilli seem to form granules at the poles suggesting polar bodies. In a few days the slide, either from the purulent fluid in the nodule, or from a skin scraping at the edge of the lesion, or from an ear clip, shows masses of acid-fast granules and cellular debris, with often an almost complete absence of the usual form of bacilli. These masses of granules tend to gather in clumps, which at times have a clear zone surrounding them.

The intensity of response to the antitoxin varies with the individual, being more severe in the more acute reaction. In approximately fifty cases treated this way, we have not found one who did not respond in the manner described. In the cases treated 
with either tetanus antitoxin or antivenom no appreciable change in the condition of the patient resulted.

Some months after the initial injection of a number of patients, as noted, we went over the records of the institution and found that most of the cases had been free from further reactions for a considerable period, also that certain individuals showed marked improvement in local skin manifestations and general condition. We therefore began treating these and other cases of reaction with smaller doses of antitoxin, repeating the injection as often as once a month if the reaction recurred. Later on we found that repeated doses gave encouraging results in several cases who were not having reactions. 2,000 units of antitoxin were given at ten day intervals. The following brief summaries of case histories are typical of the group.

\section{Case Histories}

No. I 205. Male, age 25. Duration of disease four years. Admitted to asylum June 6th, I939. Skin lesions over the body and anesthesia are shown in the photographs and charts. Laboratory findings as follows:

Urine-albumen plus I, with granular casts.

Stool-negative for parasitic ova.

Blood-W. B. C. ro, 400. R. B. C. 4,690,000 Hgb. $70 \%$.

Bacteriology-skin positive. Nasal secretions negative.

Kahn-negative.

Formol-gel-negative.

There was marked edema of the hands and legs, the hands being so stiff that the patient could use them only with difficulty. On June I 9 th, he was given 4,000 units of diphtheria antitoxin. Routine treatment with injections of chaulmoogra oil was given twice weekly, throughout the course of the treatment up to date. Diphtheria antitoxin was repeated at io day intervals, 2, ooo units being the usual dose. In all 34,000 units have been given in I4 doses. On August 5 th, there is the following notation on his chart: "Raised areas are now all flat. Many have disappeared, and some remain only as a discolouration. No raised areas remaining on face." At no time during the treatment of nearly six months has he had a leper reaction, or fever, though he states that he had many reactions before coming to the asylum.

No. I200. Male age 48 . Duration of disease 4 years. Patient was brought to the asylum by friends who believed him to be in a dying condition and wished to be rid of him before that event could take place. H is body was covered with a most serere leper reaction, with many ulcers. There was a diffuse infiltration of the entire skin with edema of hands and feet. Laboratory findings were:

Urine-heavy cloud of albumen.

Feces-negative for parasitic ova.

Blood-W. B. C. 6,450 , R. B. C. $3,630,000$. Hgb. $60 \%$.

Kahn-plus three.

Bact.- - skin plus three. nose plus two.

Formol-gel-negative.

The reactions were severe and he was not given the usual injections of chaulmoogra oil. Insteall he was given 2,00o units of diphtheria antitoxin. The beneficial results were immediate. The entire body showed scaling. The antitoxin injection was repeated at ro day intervals. On August 7 th, he came complaining of badly swollen feet and legs. The urine contained albumen three plus, which required considerable treatment, ()ctober Ioth, he was given a small dose of 
neosalvarsan. This brought on a severe reaction with fever. However after f,ooo units of antitoxin it sulsided. His general condition is much improved. This is an instance where there has been marked improvement in spite of serious complications of syphilis and nephritis. Bacteriological examinations show the characteristic fragmentation and granulation, as noted elsewhere.

No. 99. Male, age 26. Patient admitted in 1937. Duration of disease one year before admission. Stage L-1. Prognosis on examination doubtful. On December 6th, 1938, patient reported with a leper reaction. 6,000 units of antitoxin were given. A second slight reaction occurred on the $14^{\text {th }}$ of Octolser. Up to I)ecember ist, 1939, there have been no other reactions. In this case one injection effectively cleared up a reaction, and there was no recurrence for so months.

No. 868. Male, age 39. Admitted January 1936. Duration of disease r2 years. Stage I,-I, N-r. During the first six months of r939 patient had repeated reactions. July ist, 2,000 units of antitoxin were given. $\mathrm{I}_{\mathrm{P}}$ to December ist, there have been no further reactions.

No. 1047. Male, age 2r. Aclmitted June 1937. Stage I,-I. Patient was in reaction cluring all of May and first part of June. June rath, 2,000 units of antitoxin were given. Reaction occurred a month later at which time a second 2,000 units were given. Since then, after four and a half months there has been no further reaction.

No. 1 122. Male, age 32. Aclmitted Octolser 1938. Stage N-2. Duration of disease one year. Patient was given antitoxin December 6th. There have been no further reactions during the year following.

\section{EXPER IMENTS WITH DiphTheria ToxoID}

Since the effects of diphtheria antitoxin were so encouraging we decided to try diphtheria toxoid. On October 20th, I939, we selected sixty cases from the patients of the Chiengmai Leper Asylum and injected one c.c of toxoid. Cases were chosen from the following groups: L-I, L-2, L-3, N-I, N-2, N-3. Most of these cases had been under our care for a year or more and had complete case records giving the following data:-urine and stool examination, W.B.C., R.B.C., Hgb. differential count, Kahn test, formol-gel test, sedimentation rate, weight and height, blood pressure, and a careful charting of the lesions of the skin together with notations as to enlarged nerves and deformities. Some twenty cases previously treated with antitoxin were added to this group, as were a number of cases of enlarged ulnar nerves.

The patients were examined the day following injection and notation made as to where local or general reaction occurred. Within two weeks we noted that in those L (lepromatous) cases with nodules or raised areas there was first a fine scaling over, as described for the antitoxin, and then a change in the pigmentation from red to brown and a fine wrinkling or crepe-paper effect over the raised areas indicating a definite shrinking of the nodule or plaque. In the more severe skin cases the wrinkling was more marked and the vigour of the response in direct relation to the severity of the disease.

A month later the patients showed a continued improvement. The nodules and plaques were further reduced, many of them no 
longer raised, but flat with the skin. This change was present in every individual showing such lesions. (See figs I and 2). Bacteriological findings were the same as those noted in connection with antitoxin, which changes were found in every instance, excepting those cases in which no bacilli were found.

A number of the patients volunteered the information that following the toxoid their faces had become less swollen, that they "felt lighter." This was quite apparent after I4 days. There was a general reduction of the induration wherever present. In a few cases of the more severe skin types with lepromata the thickness of the skin was reduced leaving hard nodules standing out as

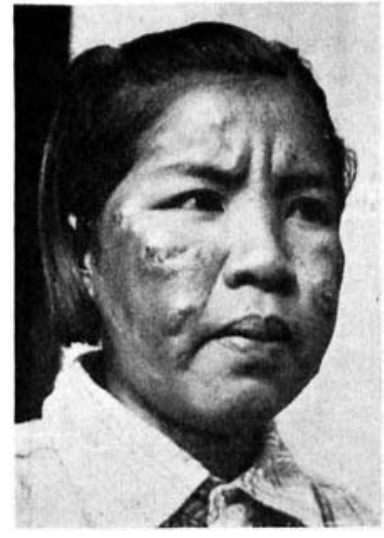

Fig. I. After treatment with toxoid. Beginning of resolution of face lesion.

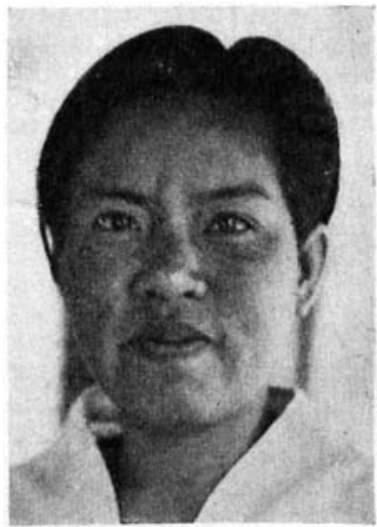

Fig. 2. Same patient four weeks later.

islands. In some few cases there was abcess formation in the centre of such nodules, which, on examination, showed granulation and cell debris with an occasional acid-fast bacillus. The granules were also acid-fast.

The effect of the treatment was less apparent in the nerve cases, but after two weeks some of the patients stated that they had experienced paresthesia of certain areas, previously anesthetic. One or two remarked that they had a complete return of sensation in previously anesthetic spots, and one patient a return of function of a partly paralyzed hand.

A month after the first injection of toxoid the group was examined for nerve enlargement and anesthesia. More than $50 \%$ showed improvement in these respects. When examined blindfolded for anesthesia, a considerable number showed definite improvement, as indicated by comparison with their previously charted lesions. Enlarged ulnar nerves in a number had returned 
to normal size and were no longer palpable. In some of these cases there was a return of sensation in part or all of the area supplied by the ulnar, together with improvement in muscular activity.

The general condition of the patients is good. A good many have gained weight. There have been only a, very few leper reactions among this group, and in those cases it has been accompanied by or caused by an attack of malaria. Only three out of eighty cases have failed to maintain the improvement gained, and they were complicated in each case by some other debilitating disease or condition.

Among the children there are several early cases in which flat depigmented areas have slowly returned to normal colour, and have all but completely disappeared, so that without a previous history one would be quite unable to make a diagnosis of leprosy.

\section{Discussion}

We believe that the granulation and fragmentation of the bacilli and the wrinkling or crepe paper appearance of the skin, seen in the advanced skin cases following treatment, is most significant. Hitherto we have always understood that such fragmentation and such wrinkling occurred only in those cases of leprosy that had acquired sufficient resistance to the disease, as to bring about a gradual diminution of all active symptoms, and that they were in all probability past the peak of their infection and were on the down grade as far as the disease was concerned. If such a stage can be induced by treatment at some early point in the disease, early recovery might be expected. The observation, that the effects of toxoid seem to be constant and that we can predict the progress with a fair certainty, is very encouraging. This is particularly true in respect to the early and as yet unmutilated cases.

In a disease of the chronicity of leprosy, observations of new methods of treatment must of necessity cover a considerable period of time. This is particularly so since this disease is noted for its long periods of exacerbation and quiescence. However this report is offered at this time in order that the results may be verified by others, and the benefits extended as widely as possible. It is hoped that further observations may confirm the impression so far obtained, that in diphtheria toxoid and antitoxin we have a new method of treatment which brings definitely beneficial results.

\section{Conclusions}

While we are not able, at present, to explain the relation between diphtheria and leprosy, or the mechanism responsible for the improvement following injections of diphtheria antitoxin and toxoid, we are convinced that such improvement does occur in a 
large percentage of cases treated. We have noted the following beneficial results from among approximately 120 cases treated:

I. Immediate reduction of leper nodules, plaques, and tuberculoids.

2. Rapid improvement in leper reactions, , with usually lasting effects.

3. Return of sensation in some previously anesthetic areas.

4. Reduction of enlarged nerves, particularly the ulnars.

5. General physical improvement.

6. Fragmentation and granulation of the bacilli.

7. As yet we have not seen any harmful or deleterious effects from this treatment.

[In a personal communication 1)r. Collier mentions that the dose of formol-toxoid used is 0.5 c.c., rising by 0.5 c.c. to 2.0 c.c., injections being given intramuscularly once a fortnight. He states that out of twelve bacteriologically positive children, seven had become negative in six months, and four more showed fewer bacilli. In a summary of his results over the period October 1939 to April I940, out of 170 cases treated with toxoid (of which 122 were of the lepromatous type) 35 became negative, 70 had fewer bacilli and 28 showed improved skin lesions. These results would appear excellent if it were not that in 147 controls treated with chaulmoogra alone ( 58 of which were of the lepromatous type) 46 became negative, 25 had fewer bacilli and 16 showed improved skin lesions. Unfortunately the subtype $\left(\mathrm{I}_{\mathrm{I}}, 2\right.$ or 3 ) of the lepromatous cases is not mentioned.-Editor.] 